\title{
Modélisation hydrodynamique et écologique des eaux côtières du golfe de Tunis
}

\author{
par R. Ben Charrada et M. Moussa
}

ENIT, Labo. Hydraulique, Tunis

\section{I 口INTRODUCTION}

Le Golfe de Tunis, situé au nord-est de la Tunisie, occupe une surface totale d'environ $1500 \mathrm{~km}^{2}$ (fig. 1). La baie de Tunis (ou le petit golfe) est une petite partie du golfe, relativement abritée, qui est située en face de la ville de Tunis et dont la surface est de l'ordre de $350 \mathrm{~km}^{2}$. Vers le sud-ouest de cette baie se trouve le complexe lagunaire formé par les deux lagunes nord et sud de Tunis qui sont séparées par le canal de navigation. Chacune de ces trois parties du complexe lagunaire communique avec la baie par un canal étroit (fig. 2). Du côté sud-ouest du golfe se trouve la ville de Tunis et ses banlieues avec une population totale de l'ordre de deux millions d'habitants.
La qualité des eaux de cet espace marin s'est dégradée au fil des dernières années suite à la pression de l'urbanisation et à l'augmentation des flux d'eaux polluées terrestres. Cette dégradation s'est traduite, surtout dans les zones côtières du golfe, par la régression des herbiers de Posidonis et l'apparition des algues nitrophiles. Tous ces aspects nous ont incité à réaliser cette étude dont le but est principalement de prévoir l'évolution de l'hydrodynamique et de la qualité des eaux littorales de ce milieu marin. Dans une première partie de cet article, nous présentons une modélisation de l'hydrodynamique du complexe lagunaire, de la baie et du golfe, et dans la deuxième partie, nous présentons une modélisation écologique de la zone côtière de la baie de Tunis en utilisant les résultats des simulations de l'hydrodynamique.

\section{Hydrodynamical and ecological modelling of Tunis bay}

A study of water quality in connection with circulation patterns in the bay of Tunis is presented. The hydrodynamics aspects were analysed using two numerical models in order to identify the internal circulation patterns. The first $1 D$ model, was designed for the lagoon of Tunis and the bay, and the second $2 D$, was applied to the gulf. The simulations showed that the water residence time in the bay varies from few days to 67 days in relation of the wind. The wind tends to produce relatively strong coastal currents. Tidal currents are rather weak and remain concentrated offshore. Then, an ecological model, describing the phytoplankton feedback in relation with the availability of the nutrients (inorganic Phosphate and Nitrogen), is proposed. Some results of ecological simulations of the littoral are presented. They showed an enrichment of the sediments with nutriments as a result of the sedimentation of dead plankton organisms. 


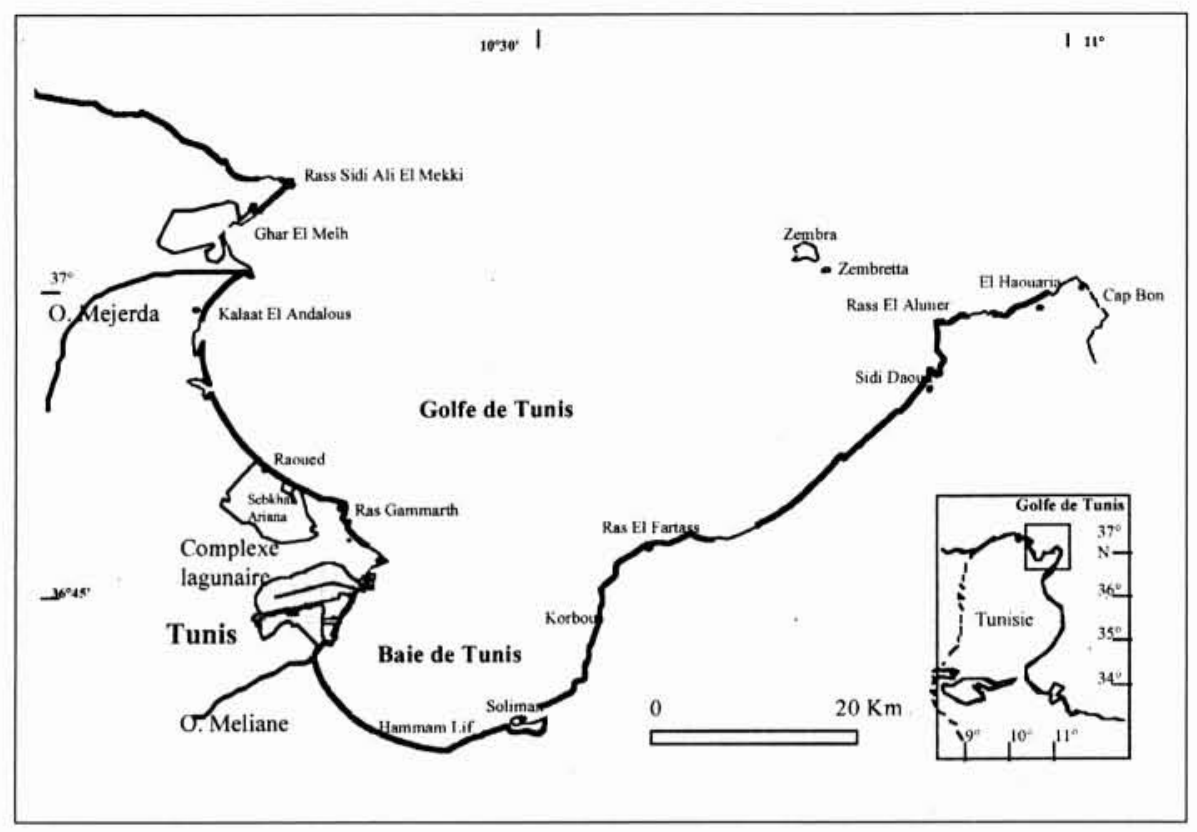

1. Situation géographique du golfe et de la baie de Tunis.

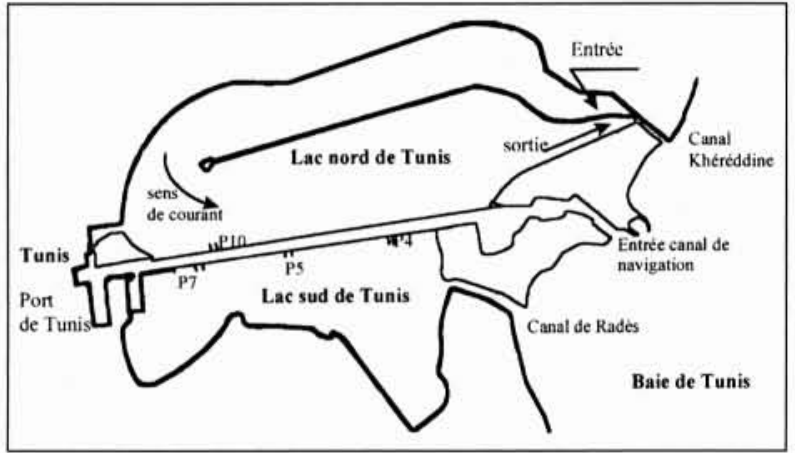

2. Le complexe lagunaire.

\section{DONNÉES GÉNÉRALES}

\subsection{Climatologie}

Le climat est caractérisé par une chaleur permanente et forte en été, une instabilité du climat pendant le reste de l'année et de faibles précipitations. Des analyses statistiques des vents, réalisées sur une période de 20 ans, montrent qu'à l'échelle saisonnière les vents ouest et nord-ouest dominent en hiver et au printemps alors que ceux du sud et du sud-est dominent en été. Les vents hivernaux, du secteur ouest, sont nettement les plus violents et soufflent plus longtemps que les autres.

\subsection{Morphologie}

Le golfe présente une grande dissymétrie géologique. D’une part, la côte occidentale, de nature alluviale, est caractérisée par des plages de sable, des vasières et des lagunes et, d'autre part, la côte orientale est d'abord constituée par un cordon littoral récent puis par une côte rocheuse avec de nombreuses falaises avec éboulis séparées par des petites criques sablonneuses. La topographie du golfe de Tunis est le résultat d'une dynamique sédimentaire particulière accentuée par les apports continentaux de l'oued Mejerda (au nord-ouest) et l'oued Meliane (au sud).
Le lac nord (fig. 2), de surface $22 \mathrm{~km}^{2}$, est partiellement divisé en deux parties par une digue : une partie nord relativement profonde $(2 \mathrm{a} 3 \mathrm{~m})$ et une partie sud de profondeur moyenne $1 \mathrm{~m}$. Ce lac est muni d'un système de régulation de la circulation d'eau à l'aide de vannes, situées dans le canal Khéréddine, et actionnées par la marée. Le lac sud, d'une surface de $15 \mathrm{~km}^{2}$, est peu profond $(0,4 \mathrm{à} 1,2 \mathrm{~m})$ et ne comporte pas de système de régulation hydraulique. Il communique avec la mer par le canal de Radès et avec le canal de navigation par trois passes qui sont équipées en pêcheries. Le canal de navigation, creusé en 1881 pour relier la baie au port de Tunis, a une longueur de $15 \mathrm{~km}$ environ, une profondeur moyenne de $8 \mathrm{~m}$ et une largeur de $100 \mathrm{~m}$.

\subsection{La marée}

Les anciennes observations, à La Goulette, indiquent une oscillation régulière, par temps calme, avec un maximum d'amplitude de $0,30 \mathrm{~m}$. Des mesures hydrographiques récentes ont été réalisées, par marégraphe, à l'entrée du lac nord, durant la période allant de juin 1989 à juillet 1990 (fig. 3). La corrélation entre ces mesures de marée et celles du vent nous a permis de faire les constatations suivantes:

- Les niveaux moyens les plus élevés (environ $0,52 \mathrm{~m}$ NGT) coïncident avec des vents nord-ouest.

- Les niveaux moyens les plus bas (de - 0,20 m NGT) ont été relevés avec des vents ouest.

- Les niveaux moyens les plus fréquents sont compris entre 0 et $0,20 \mathrm{~m}$ NGT.

- Le niveau moyen de la mer enregistré à l'entrée du lac nord est de $0,116 \mathrm{~m}$ NGT.

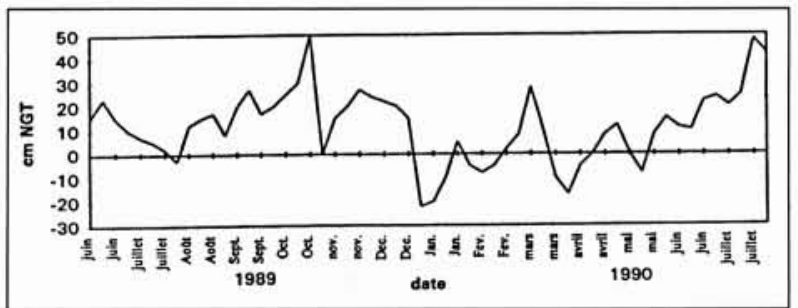

3. Enregistrements du niveau moyen de la mer. 
- A l'échelle journalière, la marée est semi-diurne (de période $12 \mathrm{~h} 24 \mathrm{mn}$ ) et son amplitude, relativement faible, varie de 0,10 à $0,30 \mathrm{~m}$ en fonction des saisons.

\section{3. — MODÉLISATION DE L'HYDRODYNA- MIQUE}

\subsection{Présentation des modèles utilisés}

Deux modèles hydrodynamiques ont été utilisés : un modèle unidimensionnel 1D, pour le complexe lagunaire, et un modèle bidimensionnel 2D, pour le golfe et la baie de Tunis. Ces modèles sont basés sur l'intégration spatiale des équations de Reynolds, pour un fluide incompressible.

\subsubsection{Modèle 1D}

Il s'agit d'un modèle intégré dans le plan vertical, perpendiculaire à l'écoulement. Les équations de ce modèle s'écrivent, en négligeant les termes de transport convectif et de dispersion :

$$
\begin{gathered}
\frac{\partial h}{\partial t}+\frac{1}{b} \frac{\partial Q}{\partial x}=-E_{v} \\
\frac{\partial Q}{\partial t}=-g b h \frac{\partial Z_{s}}{\partial x}+\frac{b}{\rho}\left(\tau_{i}-\tau\right.
\end{gathered}
$$

Dans ce système d'équations $Q$ désigne le débit, $h$, la hauteur d'eau, $b$, la largeur, $Z$, la cote de la surface libre $(h+$ $\left.Z_{f}\right), Z_{\rho}$ la cote du fond, $g$, l'accélération de la pesanteur, $\rho$, la masse volumique de l'eau, $\tau$, le frottement interfacial, $\tau_{\rho}$ le frottement au fond, $E_{v}$ l'évaporation, $t$, le temps et $X$ est l'axe principal de l'écoulement. Ces équations sont complétées par des expressions des frottements :

$\tau_{f}=\rho g Q|Q| / b^{2} h^{2} C_{h}^{2}$ et $\tau_{i}=\rho_{\text {air }} C_{i} W^{2} \cos \theta$

où $C_{h}$ est le coefficient de Chezy, $C_{i}$ le coefficient de frottement interfacial, $\rho_{\text {air }}$, la masse volumique de l'air, $W$, la vitesse du vent et $\theta$, l'angle que fait le vent avec le sens de l'écoulement.

Pour la résolution de ces équations, le domaine d'étude est discrétisé en sous-domaines élémentaires : des branches délimitées par des nœuds. Les directions des échanges entre les sous-domaines sont choisies en fonction du mode de circulation interne. Les grandeurs calculées sont, à chaque pas de temps, le débit $Q$ et le tirant d'eau $h$. Le débit est calculé dans une branche en fonction de la variation du niveau dans les deux nœuds correspondants. Le niveau est calculé dans un nœud en fonction de la somme algébrique des débits qui transitent à travers les branches de ce nœud.

Le principal avantage de ce modèle $1 \mathrm{D}$, par rapport à celui de 2D, est qu'il permet des simulations de longues durées, en vue d'un couplage avec un modèle écologique, sur une machine modeste.

\subsubsection{Modèle $2 D$}

Il s'agit d'un modèle intégré sur la hauteur. Les équations de ce modèle s'écrivent, en négligeant la dispersion [1]:

$$
\begin{gathered}
\frac{\partial h}{\partial t}+\frac{\partial h U}{\partial x}+\frac{\partial h V}{\partial y}=-E_{v} \\
\frac{\partial(h U)}{\partial t}+\frac{\partial(h U U)}{\partial x}+\frac{\partial(h U V)}{\partial y}=-g h \frac{\partial Z_{s}}{\partial x}+\frac{1}{\rho}\left(\tau_{i x}-\tau_{f x}\right)+f V h \\
\frac{\partial(h V)}{\partial t}+\frac{\partial(h V U)}{\partial x}+\frac{\partial(h V V)}{\partial y}=-g h \frac{\partial Z_{s}}{\partial y}+\frac{1}{\rho}\left(\tau_{i y}-\tau_{f y}\right)-f U h
\end{gathered}
$$

Dans ce système d'équations $U$ et $V$ sont les deux composantes de la vitesse horizontale, $\tau_{i x}$ et $\tau_{i y}$, les deux composantes du frottement interfacial, $\tau_{f x}$ et $\tau_{f y}$, les deux composantes du frottement au fond et $f$ est le paramètre de Coriolis.

La résolution de ce système d'équations est réalisée par la méthode des éléments finis, en utilisant l'algorithme d'Euler implicite couplé à la méthode de Newton Raphson [2].

\subsection{Modélisation du complexe lagunaire}

Actuellement, le complexe lagunaire constitue, pour les eaux côtières du petit golfe de Tunis, une source importante de pollution. Par la modélisation de l'hydrodynamique de ce complexe on vise principalement la quantification des flux échangés avec le golfe. Les deux lagunes (lac nord et lac sud) ont déjà fait l'objet d'études hydrodynamiques par le modèle 2D décrit ci-dessus. Ces études ont été réalisées séparément

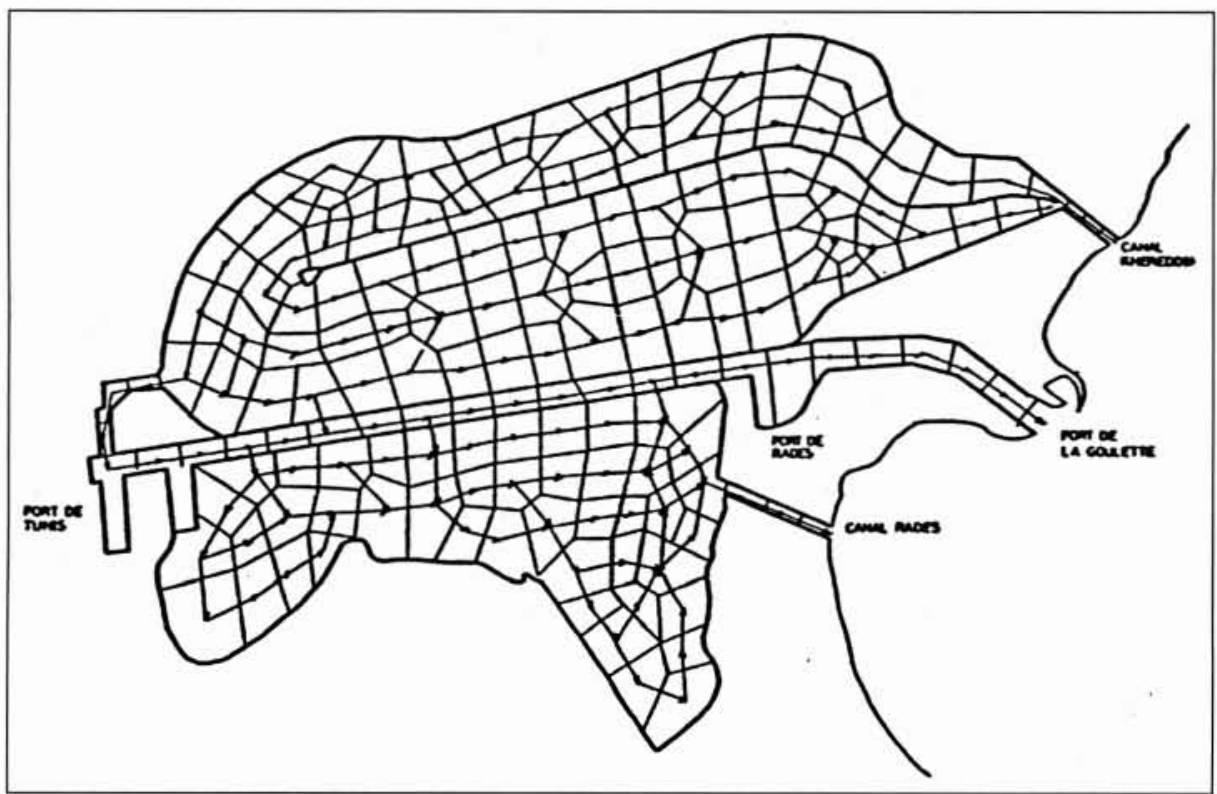

4. Discrétisation du complexe lagunaire et schématisation des circulations internes. 


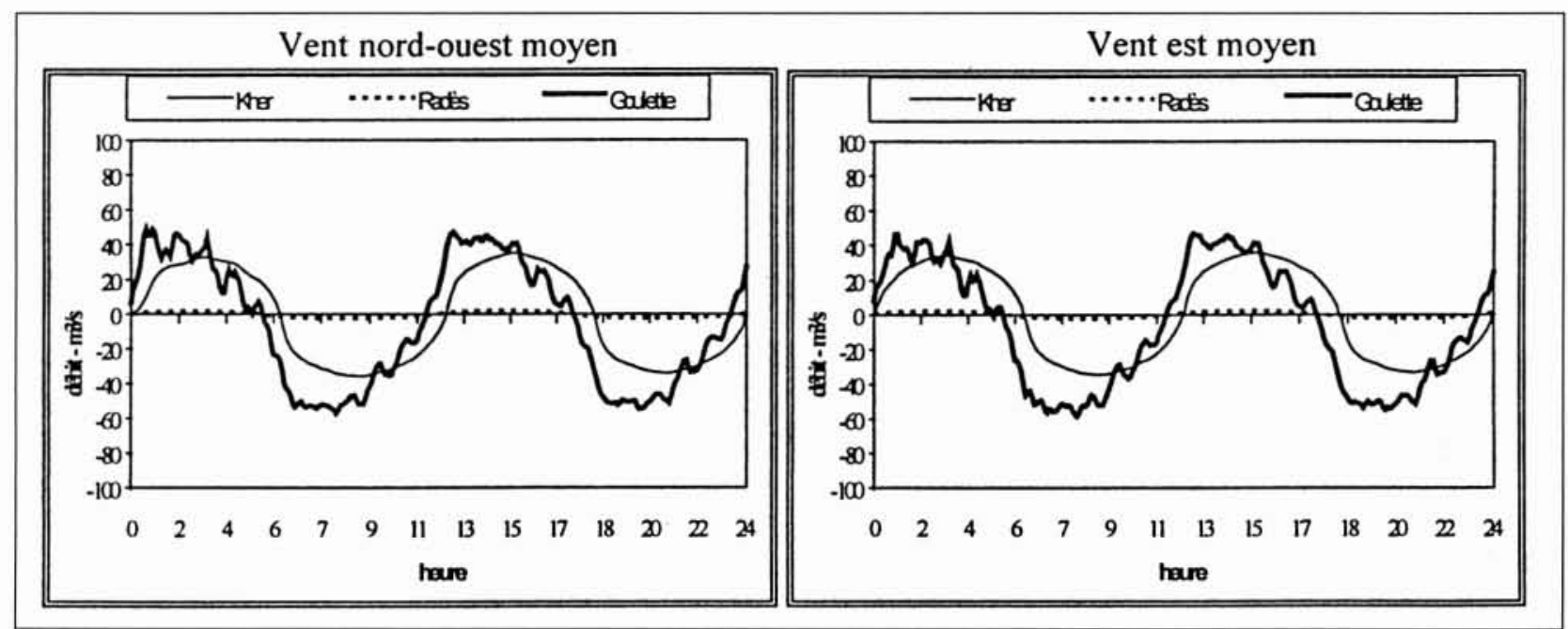

5. Echanges du complexe lagunaire avec le golfe de Tunis.

pour les deux lagunes [3] et [4]. Dans le cadre de ce travail, nous nous proposons de modéliser, simultanément, l'hydrodynamique de tout le complexe lagunaire (lac nord, lac sud et canal de navigation) à l'aide du modèle $1 \mathrm{D}$ décrit ci-dessus.

La discrétisation du complexe lagunaire en sous-domaines élémentaires (fig. 4) a été choisie en fonction des résultats des modélisations antérieures. La condition à la limite imposée, en fonction du temps, est le niveau de la marée dans la baie de Tunis. Le calage du modèle ID nous a permis de déterminer les coefficients de frottements pariétal et interfacial. Les paramètres pris en compte, pour ce calage, sont les échanges avec le golfe et le bilan total en eau pendant une période de marée.

Nous avons alors simulé l'hydrodynamique du complexe sous une condition hivernale (vent de nord-ouest de $5,9 \mathrm{~m} / \mathrm{s}$ ) et une condition estivale (vent d'est de $5,9 \mathrm{~m} / \mathrm{s}$ ), avec une marée semi-diurne d'amplitude $0,20 \mathrm{~m}$ et un niveau moyen de la mer de $0,16 \mathrm{~m}$ NGT.

L'analyse des résultats de ces simulations nous permet, pour les trois compartiments, de faire les constatations suivantes (fig. 5):

- Le lac nord: ce lac, qui communique avec le golfe par le seul canal de Khéréddine, admet un temps de séjour moyen des eaux de 21 à 33 jours suivant l'amplitude de la marée. $\mathrm{La}$ variation du niveau d'eau, au cours d'une période de marée, est faible (de $4 \mathrm{~cm}$ au maximum).

- Le lac sud: les échanges d'eau se font principalement avec le canal de navigation par les pêcheries. Les débits à travers le canal de Radès sont très faibles à cause des fortes pertes de charge. La fluctuation journalière du plan d'eau, en fonction de la marée, peut atteindre $9 \mathrm{~cm}$.

- Le canal central: la marée est transmise avec un très faible amortissement et un retard de l'ordre 20 minutes. Le canal apparaît, de ce fait, comme un milieu régulateur des échanges des deux lacs nord et sud.

\subsection{Modélisation du golfe et de la baie de Tunis}

En vue de modéliser l'hydrodynamique de la baie de Tunis, nous avons opté pour des modèles emboîtés : un modèle $2 \mathrm{D}$ de tout le golfe de Tunis qui permet de déterminer les conditions aux limites nécessaires au modèle ID de la baie.

\subsubsection{Le Golfe de Tunis}

La modélisation 2D du golfe de Tunis est réalisée avec un pas d'espace moyen de $4 \mathrm{~km}$ (fig. 6). La seule condition imposée est le niveau de la marée à la frontière nord joignant Cap Farina au Cap Bon. Le calage de ce modèle a été réalisé en se basant sur les mesures de la marée dans les trois coins du golfe tels que Cap Farina (extrême nordouest), Sidi Daoud (nord-est) et à La Goulette (au sud). Les vitesses, au niveau de la frontière nord du golfe, sont comparables aux résultats des simulations de toute la Méditerranée occidentale réalisées par LODYC à Paris VI.

Sur les figures 6 nous présentons les champs de vitesses moyennes calculées en fonction du vent. Ces simulations montrent que les courants dans le golfe de Tunis sont principalement contrôlés par le vent. En effet, par temps calme, le courant induit par la marée n'excède pas $1,5 \mathrm{~cm} / \mathrm{s}$ et reste concentré au large. Le vent génère, le long des côtes, des courants importants dont le sens dépend de la direction du vent, et au large, des courants relativement faibles.

Le bilan journalier des échanges d'eau entre la baie et le golfe de Tunis est résumé dans le tableau 1.

Nous en déduisons que le temps de séjour des eaux dans la baie de Tunis varie de 3 jours (pour des conditions très favorables à la circulation) à 67 jours (pour une faible marée et un vent calme).

Tableau 1. - Bilan journalier des échanges d'eau entre la baie et le golfe de Tunis.

\begin{tabular}{|l|c|c|}
\cline { 2 - 3 } \multicolumn{1}{c|}{} & Total entrant (en million de $\mathrm{m}^{3} / \mathrm{j}$ ) & Total sortant (en million de $\mathrm{m}^{3} / \mathrm{j}$ ) \\
\hline Vent nord-ouest de $9 \mathrm{~m} / \mathrm{s}$ & 1850 & 1843 \\
\hline Vent nord-ouest de $5,9 \mathrm{~m} / \mathrm{s}$ & 1195 & 1198 \\
\hline Vent est de $5,9 \mathrm{~m} / \mathrm{s}$ & 725 & 728 \\
\hline Vent calme & 80 & 79 \\
\hline
\end{tabular}




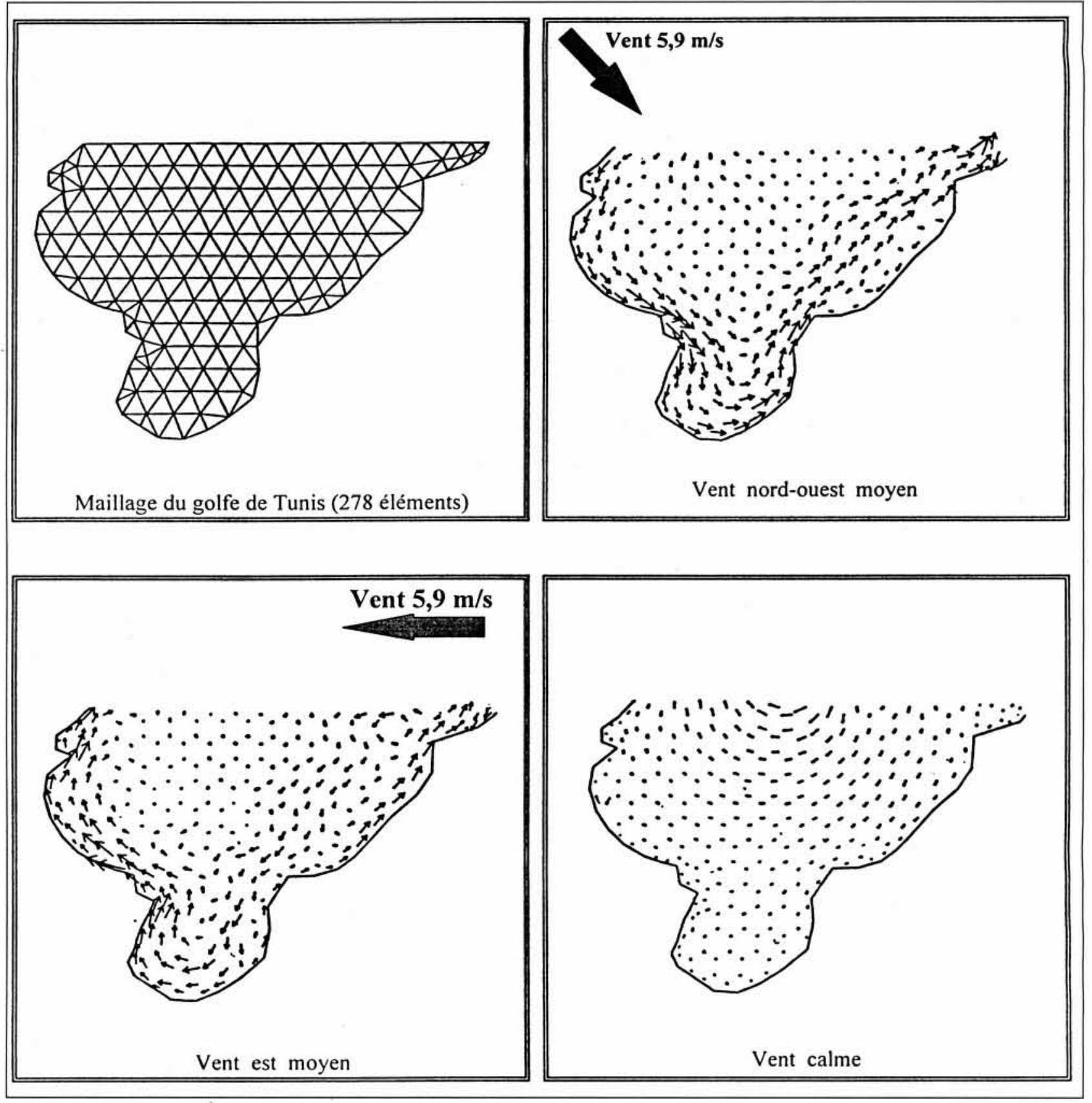

6. Maillage et champs de vitesses dans le golfe de Tunis

\subsubsection{La Baie de Tunis}

Les résultats du modèle 2D de la baie de Tunis (les circulations internes) ont été exploités pour réaliser un modèle 1D de la baie en incluant le complexe lagunaire (fig. 7). Le but de ce dernier modèle est principalement la détermination des échanges d'eau de la zone côtière qui fera l'objet d'une modélisation écologique (\$ 4.). La condition de niveau imposé à la frontière nord (joignant Cap Carthage à Ras El Fortas) provient, pour chaque scénario de simulation, des résultats du modèle $2 \mathrm{D}$ du golfe de Tunis. Le calage de ce modèle a été réalisé en se basant sur les échanges entre la baie et le golfe d'une part, et la variation du niveau de la marée au port de La Goulette d'autre part.

Les simulations par ce modèle nous ont permis de déduire le volume des échanges entre les différentes zones du domaine. Le bilan total en eau journalier de la zone côtière (zones 1, 2 et 3 - fig. 7 et 8 ), située au débouché du complexe lagunaire, varie de 155 millions de $\mathrm{m}^{3} /$ jour (pour des conditions très favorables) à environ 6 millions de $\mathrm{m}^{3} / \mathrm{jour}$ (pour une faible marée et un vent calme).

\section{MODÉLISATION ÉCOLOGIQUE DES EAUX CÔTIÈRES DE LA BAIE}

Avant de décrire le principe du modèle écologique proposé, nous présentons quelques résultats des mesures d'ordre écologique que nous avons réalisées dans la zone côtière de la baie (fig. 8). Ces mesures seront exploitées, d'une part, pour comprendre et définir la chaîne alimentaire entre les différents niveaux trophiques (Phytoplancton-Zooplancton) et, d'autre part, pour le calage du modèle écologique. 


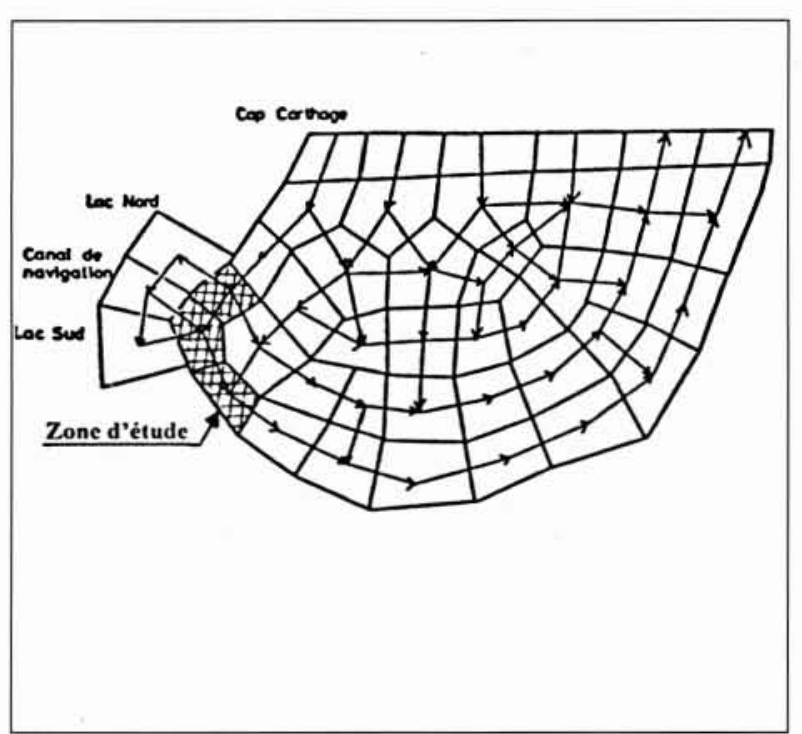

7. Schéma unifilaire de la Baie de Tunis.

\subsection{Mesures de la qualité des eaux côtières}

Deux campagnes de mesures des différentes caractéristiques de l'eau dans toute la baie de Tunis nous ont montré que la zone littorale, proche de la ville de Tunis, est la plus polluée. En effet, cette zone côtière (qui s'étend de Carthage jusqu'à Hammam-Lif) est située au débouché du complexe lagunaire et de l'Oued Meliane et reçoit actuellement la majorité des eaux polluées terrestres de la région (fig. 8).

Dans cette zone côtière, nous avons réalisé des mesures de la température de l'eau, de la salinité de l'eau, du $\mathrm{pH}$, de l'azote minéral, du phosphore minéral, de la chlorophylle- $a$ et du plancton, durant la période allant d'août 1988 jusqu'à août 1989 , avec un pas de temps de deux semaines. Les résultats de ces mesures (fig. 10) montrent que les concentrations en azote et en phosphore inorganiques dans la baie de Tunis sont généralement faibles. On peut distinguer deux périodes pendant lesquelles les concentrations sont différentes : une première période (août-décembre) où les concentrations d'azote et de phosphore sont relativement élevées alors que la concentration de la chlorophylle- $a$ est faible et une seconde période (mars-mai) pendant laquelle la situation s'inverse, ce qui correspond à la floraison printanière du phytoplancton. Au cours de cette dernière période, le phosphore minéral est épuisé et l'azote est maintenu à un niveau faible, ce qui indique une assimilation préférentielle du phosphore par le phytoplancton par rapport à l'azote.

Par ailleurs, ces mesures ont été accompagnées par une étude des fréquences d'apparition de 17 espèces de planc-

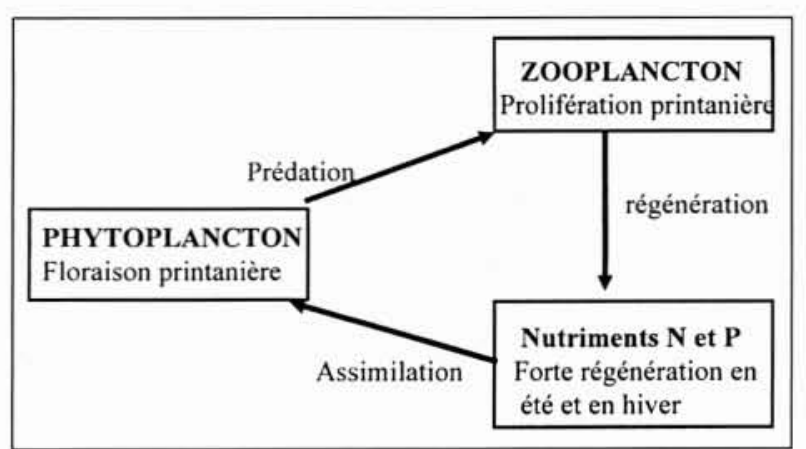

9. Schéma simplifié du cycle nutriments — plancton.

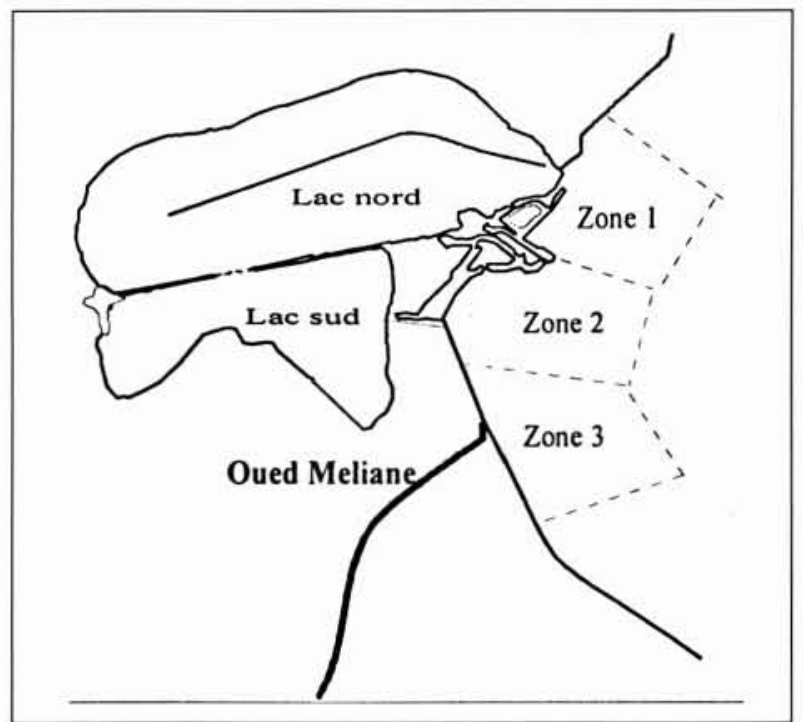

8. Situation de la zone côtière.

tontes qui comprennent notamment 4 dinoflagellés, 6 diatomées, un fagellé et 6 zooplonctontes. Les résultats ont montré qu'il y a une relation entre l'évolution saisonnière du plancton et la chlorophylle- $a$ d'une part, et de la disponibilité des nutriments d'autre part. On a remarqué que les diatomées et les dinoflagellés ont des cycles de croissance légèrement différents et qu'il y a une relation entre les deux modes de développement. Les diatomées présentent, comme la chlorophylle- $a$, deux pics annuels : le premier, le plus important, apparaît en mars-avril et le second en septembre. Chacun de ces deux pics de diatomées est suivi d'une légère augmentation du nombre des dinoflagellés et du zooplancton. Ces résultats peuvent être schématisés par la figure 9.

\subsection{Le modèle écologique}

La modélisation proposée (schématisée sur la figure 11) comporte une étude de la croissance du phytoplancton en fonction des sels nutritifs. Les lois des cinétiques utilisées sont celles de Anderson et Nival [5] complétées par les mesures in situ. Le cycle des nutriments (azote et phosphore) adopté est semblable à celui utilisé par Jorgensen ([6] et [7]).

La chaîne alimentaire s'inspire des mesures réalisées dans la zone côtère et présentées ci-dessus. Les Nutriments inorganiques dissous (azote et phosphore) sont assimilés par le phytoplancton et se transforment sous forme de nutriments organiques particulaires. Une partie du phytoplancton est digérée par le zooplancton et une seconde partie morte est transformée en matière organique détritique. Le zooplancton ingère le phytoplancton et sécrète une quantité de nutriments inorganiques dissous. La mortalité des micro-organismes (phytoplancton et zooplancton) génère de la matière organique détritique. Une partie de cette matière est minéralisée par la décomposition bactériologique pour régénérer les nutriments inorganiques dissous et le reste se dépose sur le fond. La température de l'eau agit sur la croissance du phytoplancton et sur le taux de sécrétion des nutriments par le zooplancton. Pour fermer le modèle, nous avons ajouté un troisième niveau trophique relatif aux petits poissons pélagiques dont la concentration, supposée constante, sera déterminée par calage. Les sels nutritifs (azote et phosphore inorganiques) sont régénérés soit par la minéralisation de la matière organique soit par les apports benthiques. 


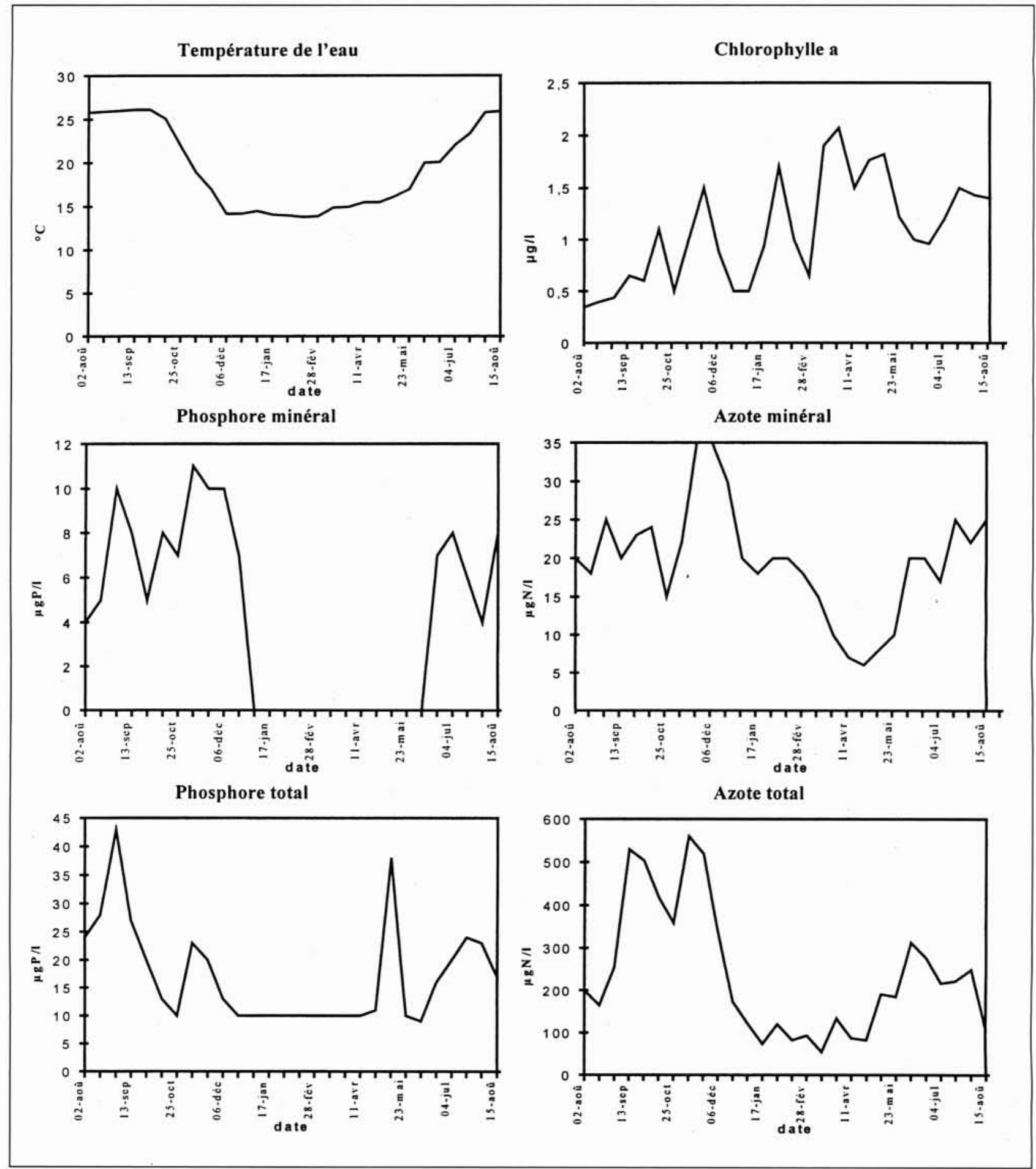

10. Résultats des mesures de la qualité des eaux dans la zone 1 (août 88-août 89).

Les lois et leurs différents coefficients utilisés sont présentés dans le tableau 2. Le système d'équations du modèle écologique est regroupé dans le tableau 3. Ce système est résolu par une méthode explicite pour les trois zones côtières représentées sur la figure 8 avec la prise en compte des échanges internes et externes. Le pas de temps adopté pour ce modèle est de 1 jour et les différents paramètres d'état sont donnés en moyennes journalières.

\subsection{Les données du modèle}

La zone côtière que l'on se propose de modéliser est représentée, dans le modèle hydrodynamique $1 \mathrm{D}$ de la baie de Tunis, par trois zones (fig. 8):

- Zone 1: située au débouché du lac nord de Tunis.

- Zone 2: située au débouché du lac sud et du canal de navigation.

- Zone 3: située au débouché de l'Oued Meliane. 


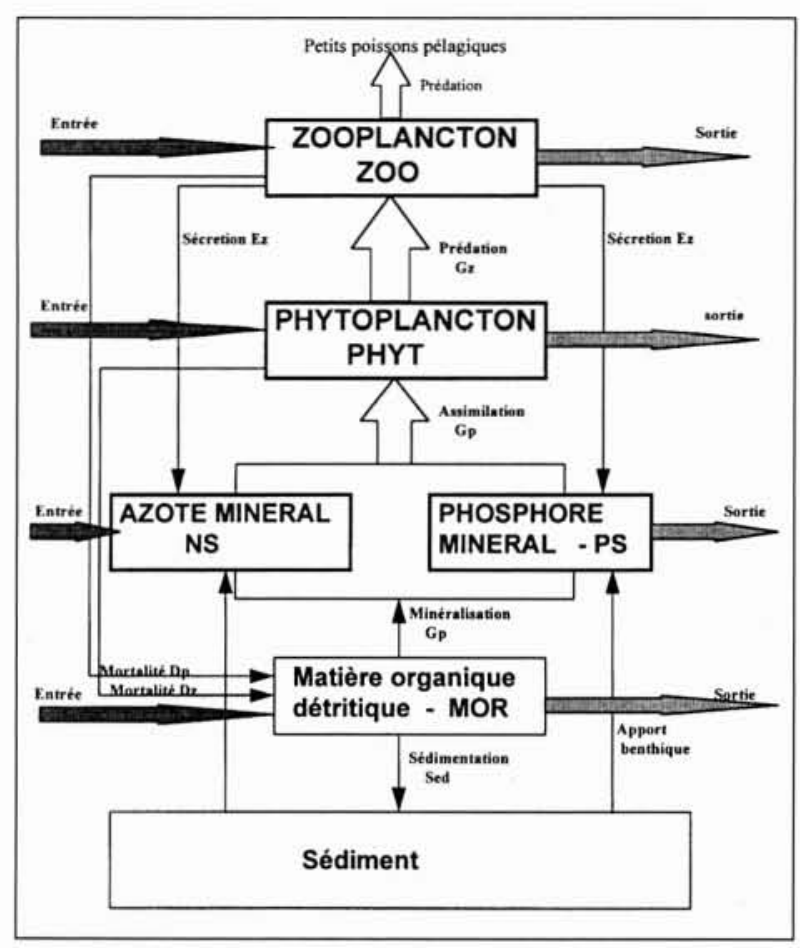

11. Schéma général du modèle écologique.

\subsubsection{Données physiques}

Elles concernent les échanges hydrodynamiques, la température de l'eau et l'éclairement solaire. Les échanges hydrodynamiques sont calculés par le modèle $1 \mathrm{D}$ présenté en $\$ 3.3$. La température de l'eau et l'éclairement solaire ont été lissés, d'après les mesures fournies par l'Institut National de la Météorologie, par les expressions suivantes :

- Température : Temp $=19,6-6 \cos [2 \pi(t-50) / 364]$ où ( $t$ en jours et Temp en ${ }^{\circ} \mathrm{C}$ ).

- Eclairement : $E=17+9 \sin [2 \pi(t-91) / 364]$ où $(t$ en jours et $E$ en $\mathrm{cal} / \mathrm{cm}^{2} / \mathrm{h}$ )

\subsubsection{Conditions aux limites}

Les conditions aux limites du modèle écologique concernent les caractéristiques de l'eau entrante dans la zone côtière : - Les apports du lac nord de Tunis dont les caractéristiques sont fournies [10].

- Les apports du canal de navigation, du lac sud et du golfe de Tunis dont les caractéristiques sont lissées à partir de quelques mesures ponctuelles.

- Le rejet de l'Oued Meliane dont la qualité est principalement celle des eaux rejetées par les stations d'épuration (Sud Meliane et Radès) et qui est fournie par l'Office de l'Assainissement.

\section{- 4.4 Résultats des simulations}

Les mesures d'août 1988 constituent les conditions initiales du modèle écologique. Les mesures de la figure 10, réalisées dans la zone 1 entre août 1988 et août 1989, ont été utilisées pour le calage du modèle. Quelques résultats de ce calage sont présentés sur les figures 12.

Nous remarquons que le modèle fournit des valeurs comparables aux mesures bien que les échanges hydrodynamiques imposés correspondent plutôt à des conditions moyennes dominantes : marée moyenne et vent constant moyen de $5,9 \mathrm{~m} / \mathrm{s}$ du secteur dominant nord-ouest.

Parmi les scénarios qui ont été simulés par le modèle, nous présentons les deux cas suivants :

- Scénario 1: Ce scénario correspond à la situation actuelle des rejets terrestres dans la baie de Tunis. L'apport en nutriments de l'Oued Meliane représente le rejet actuel de la station d'épuration sud Meliane (soit $8700 \mathrm{~m}^{3} / \mathrm{s}$ ). Le sens de circulation dans la zone côtière (schématisé sur la figure 13) ainsi que les échanges hydrodynamiques sont donnés par le modèle $1 \mathrm{D}$ avec des conditions dominantes : marée moyenne et vent constant moyen du secteur nordouest. Quelques résultats de simulation de ce scénario, pour une période de 10 ans, incluant l'année de calage, et correspondant à la zone 1, sont présentés sur les figures 15 .

- Scénario 2: Ce scénario consiste à prévoir le comportement de l'écosystème en cas d'augmentation des flux urbains. L'apport de l'Oued Meliane dans la zone 3 représente le rejet de la future station d'épuration sud Meliane

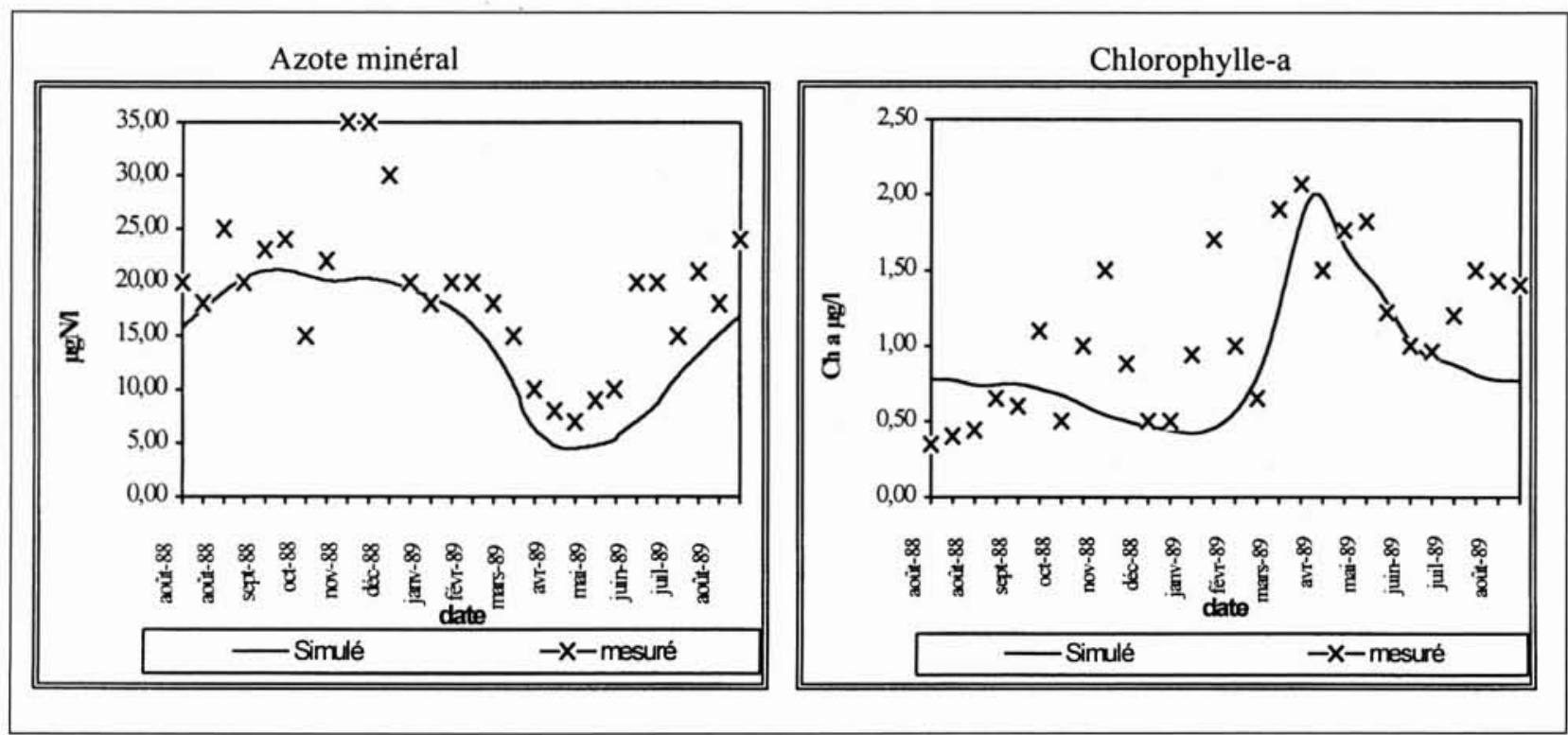

12. Résultats de calage du modèle écologique. 
Tableau 2. - Lois et coefficients utilisés.

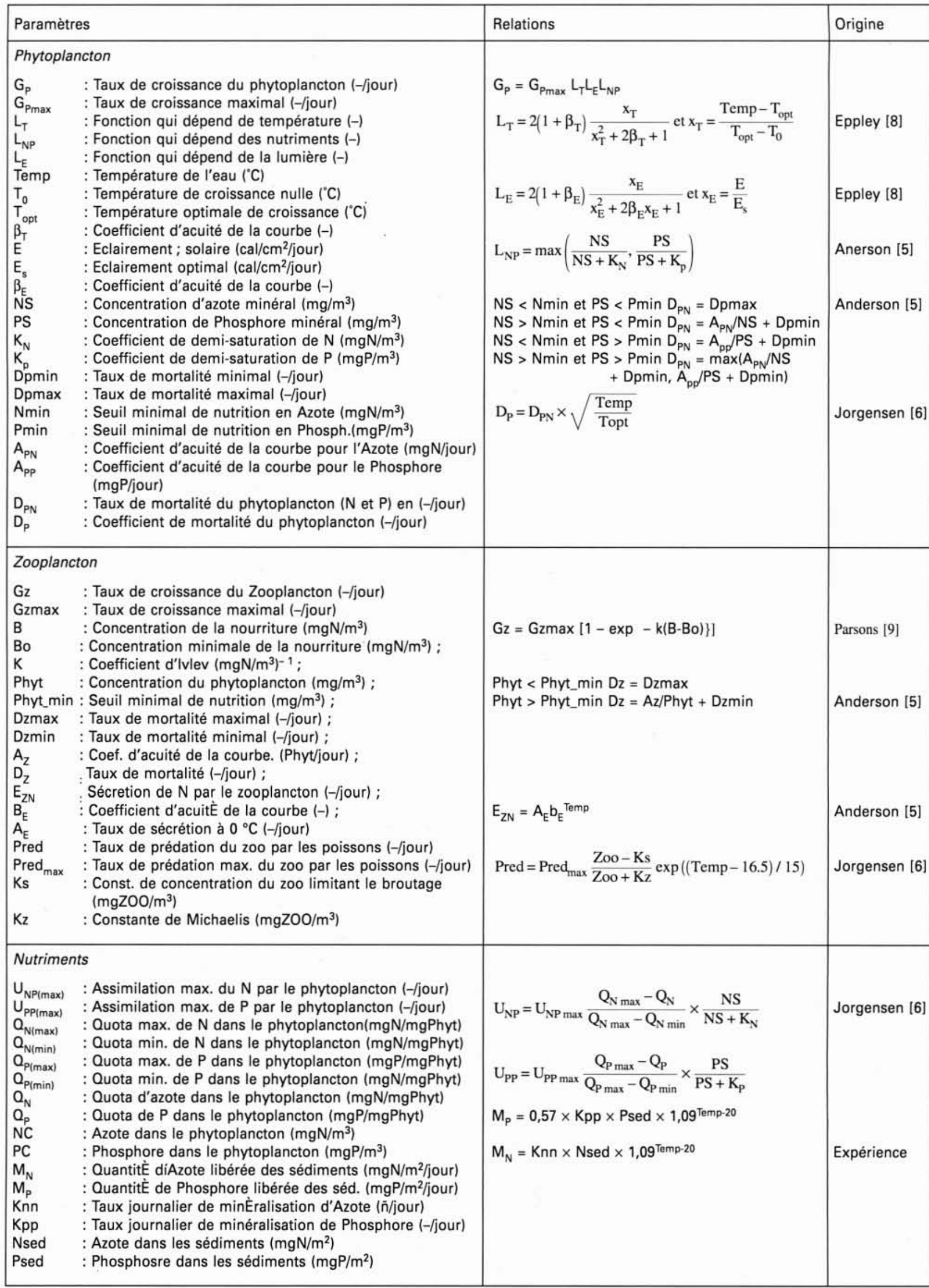


Tableau 3. : Système d'équations du modèle écologique (présenté en terme de variation journalière).

\begin{tabular}{|c|c|c|}
\hline Equations & & Paramètres \\
\hline $\begin{array}{l}d Z O O=\left(G_{Z}-D_{Z}-E_{Z N}-\frac{Q}{V}\right) Z O O-\frac{P r e d}{Y} \times \text { Poisson }+d Z O O_{i n} \\
d P H Y T=\left(G_{P}-D_{P}-\frac{Q}{V}\right) P H Y T-G_{Z} Z O O+d P H Y T_{i n} \\
d M O R=D_{P} P H Y T+D_{Z} Z O O-\left(M I N+S E D+\frac{Q}{V}\right) M O R+d M_{\text {in }} \\
d N S=E_{Z N} N Z-G_{P} N C-\frac{Q}{V} N S+M I N N D+d N S_{i n}+M_{N} / H \\
d P S=E_{Z N} P Z-G_{P} P C-\frac{Q}{V} P S+M I N P D+d P S_{i n}+M_{P} / H \\
d N C=U_{N P} P H Y T-\left(D_{P}+\frac{Q}{V}\right) N C-G_{Z} N Z+d N C_{i n} \\
d N D=D_{P} N C+D_{Z} N Z-\left(M I N+S E D+\frac{Q}{V}\right) N D+d N D_{i n} \\
d P C=U_{P P} P H Y T-\left(D_{P}+\frac{Q}{V}\right) P C-G_{Z} P Z+d P C_{i n} \\
d P D=D_{P} P C+D_{Z} P Z-\left(M I N+S E D+\frac{Q}{V}\right) P D+d P D_{i n} \\
d P s e d=M i n \times P D \times H-M_{P} \\
d N s e d=M i n \times N D \times H-M_{N} \\
d F_{N Z}=G_{Z}\left(Q_{N}-F_{N Z}\right) \\
d F_{P Z}=G_{Z}\left(Q_{P}-F_{P}\right) \\
d F_{N F}=\frac{P r e d}{Y}\left(F_{N Z}-F_{N F}\right) \\
d F_{P F}=\frac{P r e d}{Y}\left(F_{P Z}-F_{P F}\right)\end{array}$ & $\begin{array}{l}\mathrm{F}_{\mathrm{NZ}} \\
\mathrm{F}_{\mathrm{PZ}} \\
\mathrm{F}_{\mathrm{NF}} \\
\mathrm{F}_{\mathrm{PF}} \\
\text { Poisson } \\
\text { MIN } \\
\text { SED }\end{array}$ & 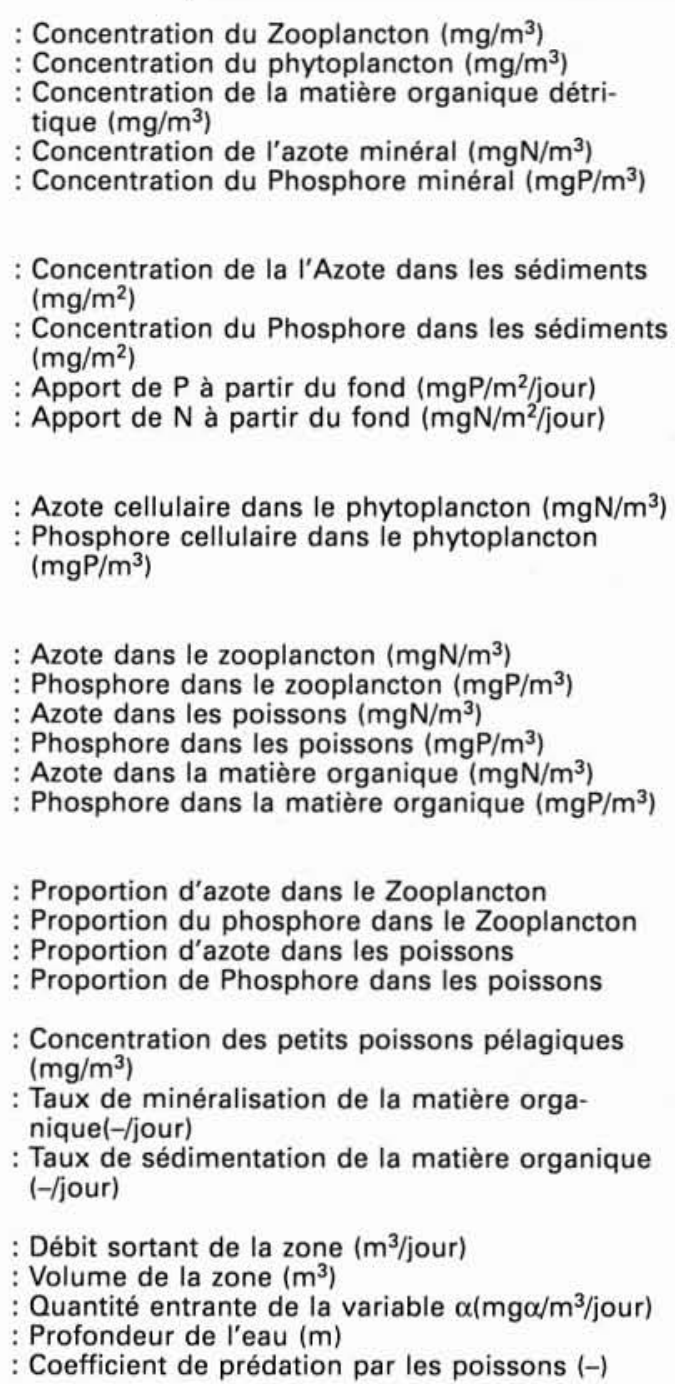 \\
\hline
\end{tabular}

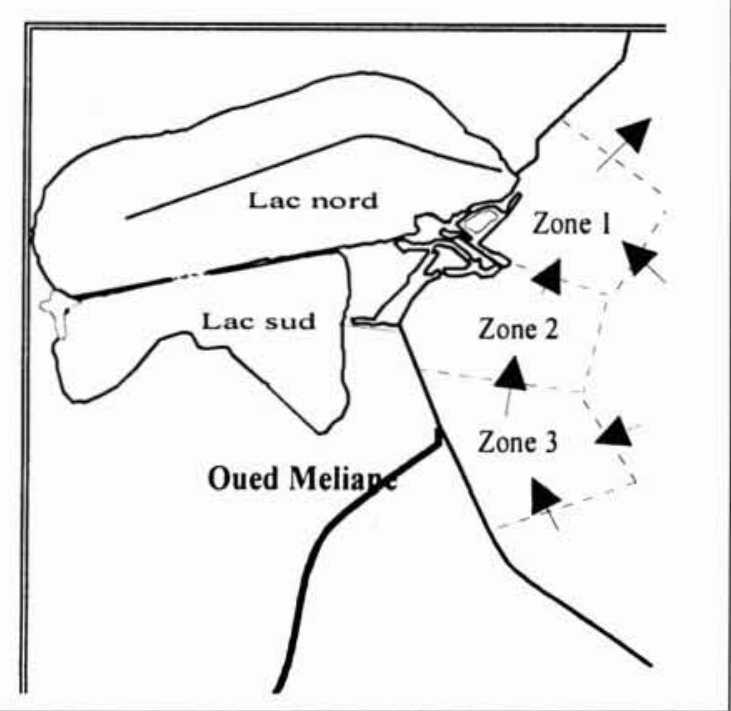

13. Sens de circulation du scénario 1.

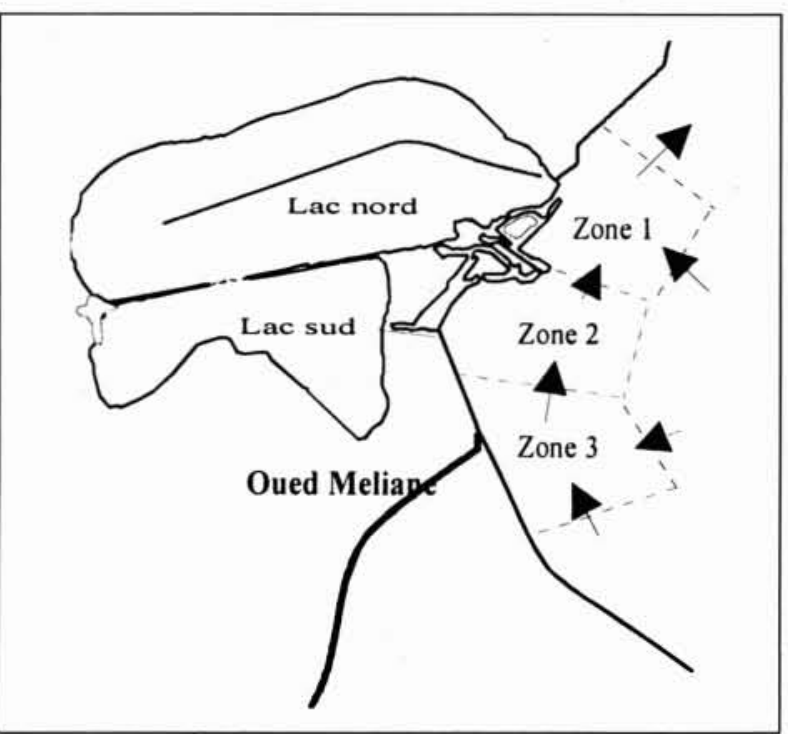

14. Sens de circulation du scénario 2. 


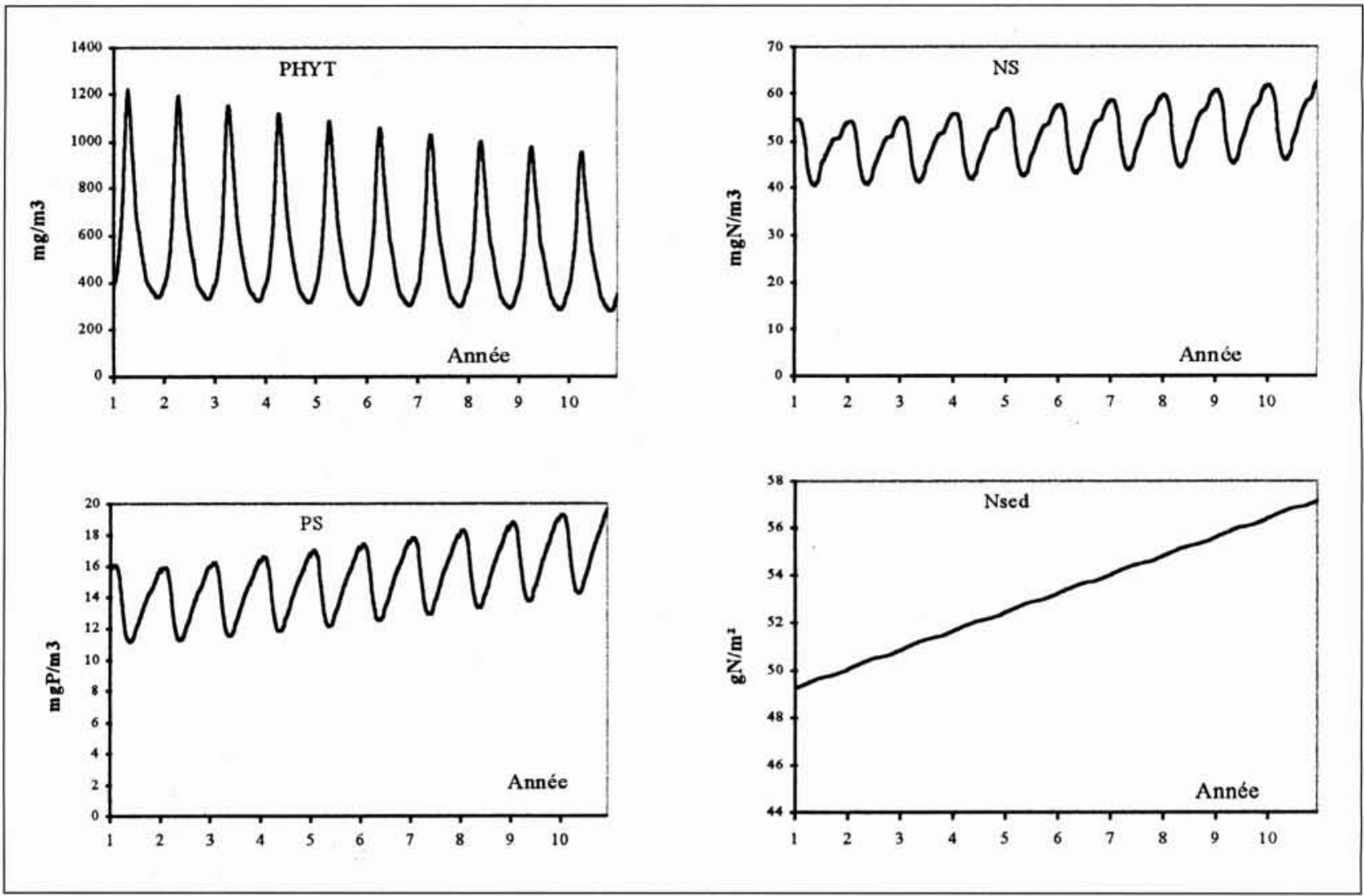

15. Résultats des simulations (scénario 1). Phyt: Phytoplancton, NS : Azote soluble dans l'eau, PS : Phosphore soluble dans l'eau, Nsed : Azote dans les sédiments.

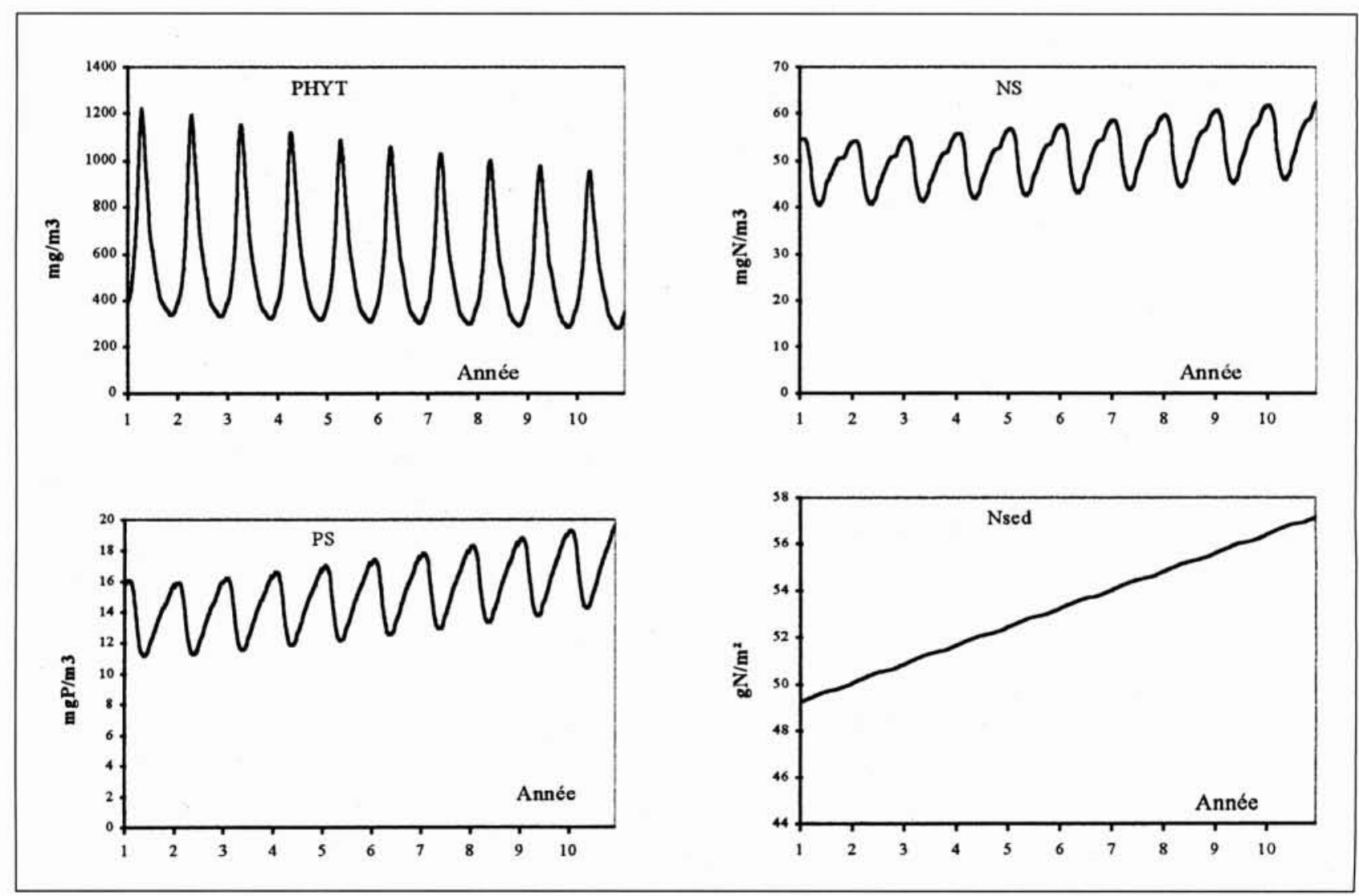

16. Résultats des simulations (scénario 2). Phyt: Phytoplancton, NS : Azote soluble dans l'eau, PS : Phosphore soluble dans l'eau, Nsed : Azote dans les sédiments. 
planifiée pour l'an 2010 (soit $59200 \mathrm{~m}^{3} / \mathrm{s}$ ). Le sens de circulation dans la zone côtière (schématisé sur la figure 14) ainsi que les échanges hydrodynamiques sont donnés par le modèle 1D avec les conditions fictives suivantes : marée moyenne et vent constant moyen du secteur est, défavorable à la dispersion de la pollution vers le large. Quelques résultats de simulation de ce scénario, pour une période de 10 ans après une année de stabilisation du comportement de l'écosystème, correspondant à la zone 1 , sont présentés sur les figures 16.

\subsection{Analyse des résultats}

Les résultats des simulations montrent une augmentation des sels nutritifs dissous, dans l'eau et dans les sédiments. Pour le scénario 1 , la concentration des sels nutritifs croît en allant de la zone 1 vers la zone 3, et la situation s'inverse pour le scénario 2. Cette migration des nutriments est la conséquence directe de la circulation des eaux.

L'augmentation des sels nutritifs est accompagnée par une diminution de la concentration du phytoplancton dans le milieu. La concentration d'azote dans les sédiments est plus importante que celle du phosphore. L'enrichissement des sédiments en nutriments organiques est dû à la sédimentation des particules mortes, de phytoplancton et de zooplancton. - Les nutriments inorganiques dans l'eau: les variations absolues des concentrations par rapport aux valeurs initiales, après 10 ans, sont indiquées dans le tableau 4. L'augmentation de ces concentrations est due aux rejets terrestres d'une part, et aux libérations à partir des sédiments d'autre part.

La variation de l'azote et du phosphore inorganique dissous dans l'eau, à l'échelle annuelle, montre que ces deux paramètres d'état ont des cycles comparables. Les moyennes annuelles sont respectivement de $14,4 \mathrm{mgN} / \mathrm{m}^{3}$ et de $5,6 \mathrm{mgP} / \mathrm{m}^{3}$ avec des maxima de l'ordre de $22 \mathrm{mgN} / \mathrm{m}^{3}$ et de $7,8 \mathrm{mgP} / \mathrm{m}^{3}$ pour le scénario 1 . La corrélation avec le cycle du phytoplancton montre, comme pour les mesures in situ, qu'il y a une étroite relation entre les deux modes de variation saisonnière. On enregistre pendant l'été et l'automne les valeurs de nutriments les plus élevées lorsque la concentration du phytoplancton est faible. Au printemps, ces nutriments sont assimilés par le phytoplancton et leurs concentrations dans l'eau, deviennent très faibles. Les résultats montrent que le rapport trophique NIP (en équivalent atomique) varie entre 4,5 et 7 avec une moyenne annuelle de 6 , ce qui traduit une assimilation de ces deux formes des nutriments $(N$ et $P$ ) par le phytoplancton dans des proportions peu différentes [11]. Les fortes teneurs de nutriments obtenues pour le scénario 2 sont essentiellement dues à l'impact du rejet de l'oued Meliane qui a été estimé pour l'horizon 2010. - Les nutriments dans les sédiments : l'enrichissement des sédiments en nutriments (organiques et inorganiques), dans la couche supérieure d'épaisseur $5 \mathrm{~cm}$, est d'environ $16 \%$ en azote et $7,4 \%$ en phosphore, par rapport à la situation initiale (tabl. 4). En période estivale, la libération des nutriments inorganiques dans l'eau est légèrement supérieure à la sédi- mentation des particules mortes à cause des hautes températures. Pendant le reste de l'année, ce phénomène s'inverse et le bilan total annuel montre que la sédimentation des nutriments est supérieure à la libération. Cette différence est due au fait que le relargage s'effectue à partir de l'eau interstitielle, après décomposition bactérienne des nutriments organiques, alors que le phénomène de sédimentation est purement physique et ne dépend que de l'hydrodynamique du milieu et de la concentration des particules mortes dans l'eau. - Le Phytoplancton: les résultats des simulations, pour une période de dix ans, montrent que le phytoplancton présente un comportement stable et une variation comparable aux mesures : un pic printanier prononcé (de l'ordre de $1000 \mu \mathrm{g} / \mathrm{l}$ pour le scénario 1) qui coïncide avec les conditions optimales de croissance (température et éclairement solaire), un second pic moins prononcé à la fin du mois de septembre et partout ailleurs une concentration qui varie de 300 à $500 \mu \mathrm{g} / \mathrm{l}$ (fig. 17).

Une baisse de la concentration moyenne annuelle du phytoplancton est observée au fur et à mesure que le milieu s'enrichit en nutriments. Cette situation a été aussi observée par Aubert [12] qui montre, à partir de mesures en mer, que des taux élevés des sels nutritifs n'entraîneraient pas forcément des taux élevés du phytoplancton. En effet, le phytoplancton est tributaire du niveau supérieur relatif au zooplancton dont la croissance est activée par des hautes concentrations du phytoplancton. C'est pourquoi nous avons observé deux pics annuels différents du zooplancton (fig. 17) : le premier $\left(1948 \mathrm{mg} / \mathrm{m}^{3}\right.$ pour le scénario 1) apparaît à la fin de la première semaine du mois d'avril, soit deux semaines après le pic du phytoplancton, et le second (moins important : $940 \mathrm{mg} / \mathrm{m}^{3}$ pour le scénario 1) au début du mois d'octobre (soit quatre semaines après le second pic du phytoplancton). L'enrichissement du milieu en sels nutritifs agit donc directement sur la croissance du phytoplancton et indirectement sur celle du zooplancton qui régénère de fortes concentrations des nutriments. Cela traduit le phénomène de l'eutrophisation qui a été étudié par plusieurs auteurs dans d'autres milieux Méditerranéens [13].

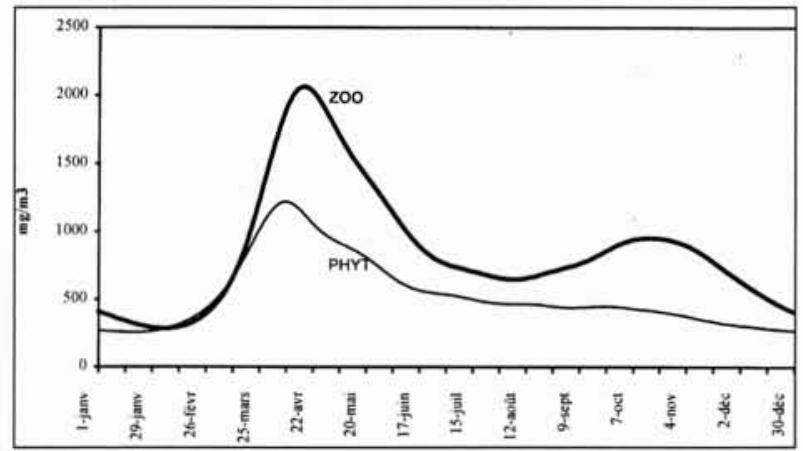

17. Variation saisonnière du zooplancton et du phytoplancton (Zone 1 et scénario 1).

Tableau 4. - Variation des concentrations moyennes en nutriments après 10 ans.

\begin{tabular}{|c|c|c|c|c|c|c|c|}
\hline \multicolumn{2}{|c|}{} & \multicolumn{3}{c|}{ Azote - N inorganique } & \multicolumn{2}{c|}{ Phosphore - P inorganique } \\
\hline & & Zone 1 & Zone 2 & Zone 3 & Zone 1 & Zone 2 & Zone 3 \\
\hline \multirow{2}{*}{$\begin{array}{c}\text { Eau } \\
\left(\mathrm{mg} / \mathrm{m}^{3}\right)\end{array}$} & Scénario 1 & $+1,5$ & $+5,7$ & $+18,2$ & $+0,5$ & $+1,6$ & $+4,3$ \\
\cline { 2 - 8 } & Scénario 2 & +40 & $+34,4$ & $+31,6$ & +11 & +10 & $+9,4$ \\
\hline \multirow{2}{*}{$\begin{array}{c}\text { Sédiment } \\
\left(\mathrm{g} / \mathrm{m}^{2}\right)\end{array}$} & Scénario 1 & $+3,8$ & $+5,9$ & $+7,9$ & $+0,8$ & $+0,08$ & $+0,07$ \\
\cline { 2 - 8 } & Scénario 2 & $+7,9$ & $+5,8$ & $+2,5$ & +1 & $+0,22$ & $+0,13$ \\
\hline
\end{tabular}




\section{$\mathrm{V} \square \mathrm{CONCLUSIONS}$}

Un modèle hydrodynamique de la baie de Tunis a été développé tenant compte des courants dans le golfe ainsi que des échanges avec le complexe lagunaire. En se basant sur des mesures in situ, un modèle écologique de la zone côtière a été mis au point. Il rend bien compte des évolutions de la qualité de l'eau et constitue ainsi un outil pour l'étude de l'écosystème côtier. Des mesures complémentaires permettront d'améliorer ce modèle et surtout de traiter l'écologie de toute la baie de Tunis. Il serait également intéressant de prévoir le comportement écologique de la zone côtière sous l'effet de changement de vent, en direction et en intensité, avec différentes périodes.

\section{REMERCIEMENTS}

Les auteurs tiennent à remercier bien vivement Messieurs L. Masbernat et C. Thirriot, Professeurs à l'I.N.P. de Toulouse, pour leurs conseils et critiques judicieuses de ce travail.

\section{RÉFÉRENCES}

[1] Moussa M. - Turbulence et circulations générées par le vent dans les systèmes aquatiques peu profonds - Application au lac de Tunis. Thèse de Doctorat de l'INP Toulouse, 1986.

[2] LINE A. - Notice d'utilisation du programme MEFAME. Rap. interne IMF Toulouse $\mathrm{N}^{\circ} 249,1987$.
[3] Ben Charrada R., Line A., Masbernat L., Moussa M. Impact des aménagements sur l'hydrodynamique du lac nord de Tunis. Modèle aux éléments finis. $2^{\mathrm{c}}$ Colloque Maghrébin sur les "Modèles Numériques de l'Ingénieur ". Rabat (Maroc), Nov. 1989.

[4] Moussa M., Ben Charrada R. - Modélisation hydrodynamique du lac sud de Tunis. Rap. interne ENI Tunis, 1991.

[5] Anderson V., Nival. P. - Modèle d'écosystème pélagique des eaux côtières de la Mer Ligure. Oceanologica Acta, $\mathrm{N}^{\circ}$ Sp. 211-217, 1988.

[6] JORGENSEN S.E. - An Eutrophication model for a lake. Ecol. modelling, 2, pp. 147-165, 1976.

[7] JORGENSEN S.E. et al. - Ecological Buffer Capacity. Ecol. modelling, 3, pp. 39-61, 1977.

[8] EPPLEY R.W. - Temperature and phytoplankton growth in the sea. Fish. Bull., 70, pp. 1063-1085, 1972.

[9] PARSONS T. et al. - Some observations on the dependance of zooplankton grazing on cell size and concentration of phytoplankton blooms. J. Oceanogr. Soc. Jpn, 23, pp. 10-17, 1967.

[10] Ben Slimane A., Masbernat L., Moussa M. - Modèle hydro-écologique du lac de Tunis. La Houille Blanche, $\mathrm{N}^{\circ} 3 / 4$, pp. 287-292, 1990.

[11] Chiaudani G., Gaggio G.F. et Vighi M. - Synoptic survey of the distribution of nutrients in italian adriatic coastal water. Thalassia Jugoslavia 19: 77-86, 1983.

[12] AUBERT M. - Eutrophie et dystrophie en milieu marin. Phénomènes planctoniques et bactériens. Revue Int. d'Océan. Médicale du CERBOM-Nice, Tomes L33 et L34, 1986.

[13] Ben CharRada R. - Impacts des aménagements de restauration sur la qualité des eaux et des peuplements benthiques du lac nord de Tunis. Mar. Life, vol. 5(1), pp. 51-64, 1995. 